\title{
Pratiques d'élevage et qualité nutritionnelle des lipides des poissons
}

\author{
Françoise MÉDALE \\ Inra, \\ UMR nutrition aquaculture et génomique, \\ Pôle hydrobiologie Inra, \\ 64310 Saint-Pée-sur-Nivelle, \\ France \\ <medale@st-pee.inra.fr>
}

\begin{abstract}
Fish lipids contain a higher proportion of $n-3$ long chain highly unsaturated fatty acids (LC HUFA) than other animals. Fish is the primary source of $n-3$ LC-HUFA such as eicosapentaenoï acid (EPA C20:5 n-3) and docosahexaenoï acid (DHA C22:6n-3) in human food. Because of limitations of fisheries capture, the supply of fish market with products from fish farming is growing. Unlike fisheries products, fish from farming can be tailored through rearing practices. Changes in n-3 LC-HUFA of fish flesh can be achieved either directly by feeding fish with diets rich in fish oil or indirectly by increasing muscle fat content through feeding manipulations (ration size, feed energy content) and genetic selection. The addition of vitamin $E$ as an antioxidant in fish feed increases the levels of vitamin $E$ in muscle, which prevents LC-HUFA from peroxidation.
\end{abstract}

Key words: fish, feeding, $n-3$ fatty acid, genetic selection, lipid peroxidation
Les lipides de la chair des poissons se caractérisent par leur richesse en acides gras polyinsaturés à longue chaîne (AGPI-LC) de la série oméga-3 ou n-3, en particulier l'acide eicosapentaénoïque (C20:5 n-3 ou EPA) et I'acide docosahexaénoïque (C22:6 n-3 ou DHA). $L^{\prime}$ abondance de ces acides gras (AG) à longue chaîne n-3 est une particularité du monde aquatique. Le phytoplancton, à la base de la chaîne trophique des animaux aquatiques, possède (à l'exception des chlorophycées) l'équipement enzymatique nécessaire à la synthèse $d$ 'acide alphalinolénique (C18:3 n-3), précurseur de I'EPA et du DHA. Ces AG sont ensuite transférés par la voie alimentaire aux étages supérieurs de la chaîne trophique, zooplancton puis crustacés, mollusques et poissons dont la composition en AG de la chair reflète celle des lipides de leur nourriture. Les AGPI-LC n-3 sont les constituants majeurs des phospholipides où ils jouent un rôle primordial dans le maintien de la fluidité membranaire. Ils sont particulièrement importants chez les animaux poïkilothermes - dont la température corporelle est identique à celle du milieu - pour maintenir la fonctionnalité des membranes, même à basse température. $D^{\prime}$ après Crockett (2008), leur concentration dans les phospholipides des membranes cellulaires est inversement proportionnelle à la température de vie des animaux. On trouve aussi les AGPI-LC dans les triglycérides de réserve, lipides neutres qui constituent une forme de stockage d'énergie facilement mobilisable. L'augmentation des réserves énergétiques musculaires sous forme de lipides se traduit donc par une augmentation du contenu en EPA et en DHA de la chair des poissons.
Les poissons sont la principale source d'AGPILC n-3 dans l'alimentation humaine. La demande en poisson pour la consommation humaine est en constante progression, en raison de la croissance de la population mondiale. Or, depuis plus de 20 ans, les captures de pêches stagnent autour de 85-90 millions de tonnes par an (FAO, 2008) dont environ deux tiers sont disponibles pour la consommation humaine. Le développement de l'élevage est donc nécessaire pour répondre à la demande. Au cours des 15 dernières années, la production aquacole mondiale s'est accrue de plus de $8 \%$ par an. Actuellement, près de $50 \%$ des produits aquatiques consommés dans le monde proviennent de l'élevage (FAO, 2008). En France, cette proportion n'est que de $15 \%$ mais tend à augmenter d'année en année (FranceAgrimer). Outre la régularité des approvisionnements, la traçabilité et la fraîcheur, I'un des atouts de l'aquaculture est la possibilité de moduler la composition de la chair des poissons par les pratiques d'élevage. Le muscle des poissons présente une grande plasticité tant pour sa teneur en lipides que pour sa composition en AG. Cet article décrit les différentes stratégies permettant d'optimiser la teneur en AGPI-LC n-3 de la chair des poissons par les pratiques d'élevage: augmentation de la teneur en lipides du muscle par le taux d'alimentation, le contenu énergétique de l'aliment ou la sélection génétique, dans les limites permises par l'espèce, modification de la composition en $\mathrm{AG}$ en fonction de la nature des huiles du régime alimentaire, prévention de la dégradation des acides gras polyinsaturés (AGPI) par peroxydation grâce à l'apport alimentaire d'antioxydants.

\section{Effet de I'alimentation sur la teneur en lipides de la chair des poissons}

Le taux d'alimentation (taille de la ration) et le contenu énergétique de l'aliment sont les moyens les plus efficaces pour moduler la teneur en lipides de la chair des poissons d'élevage. Les lipides étant plus concentrés en énergie que les glucides $(39,5 \mathrm{~kJ} / \mathrm{g}$ lipide contre $17,2 \mathrm{~kJ} / \mathrm{g}$ glucide) et mieux digérés par les poissons d'eau froide, l'augmentation du contenu en énergie digestible des aliments aquacoles est généralement réalisée par adjonction de lipides. L'augmentation de l'apport énergétique alimentaire (via la quantité d'aliment distribuée ou le contenu énergétique de l'aliment) conduit, chez pratiquement toutes les espèces, à une augmentation des lipides corporels accompagnée d'une diminution de la teneur en eau (Corraze et Kaushik, 1999). Cependant, on observe de grandes disparités entre espèces quant aux compartiments corporels dans lesquels sont stockés les lipides.

Les poissons constituent des réserves énergétiques sous forme de dépôts de lipides dans différents endroits du corps : sous la peau (tissu adipeux sous-cutané), dans la cavité abdominale autour du tube digestif (tissu adipeux périviscéral), dans le foie, le cerveau, le muscle rouge et le muscle squelettique blanc où la majorité des adipocytes sont dispersés entre les fibres musculaires et sont particulièrement abondants au sein des cloisons de tissu conjonctif séparant les feuillets musculaires (Henderson et Tocher, 1987). L'importance relative des dépôts lipidiques dans ces différents sites est caractéristique de l'espèce considérée. Par exemple, le foie est un 
site majeur de stockage des lipides chez la morue comme chez beaucoup d'autres poissons marins, alors que le tissu adipeux périviscéral est le site de stockage prédominant chez les salmonidés comme la truite. La capacité des tissus musculaires à stocker des lipides est à la base $d^{\prime}$ une classification qui distingue des espèces maigres (moins de $2 \%$ de lipides dans le muscle), des espèces grasses (teneurs en lipides dans le muscle supérieures à 8-10\%) et des espèces dites « intermédiaires » qui déposent les lipides dans le muscle ( 2 à $8 \mathrm{~g} / 100 \mathrm{~g}$ ) et dans d'autres sites tels que le tissu adipeux périviscéral comme c'est le cas chez la truite (Médale, 2009). Les résultats de différentes études (Regost et al., 2001 ; Santinha et al., 1999 ; Gélineau et al., 2001) rapportés dans le tableau 1 montrent que l'accroissement des dépôts de lipides résultant d'une augmentation de l'apport énergétique alimentaire concerne principalement les sites préférentiels de stockage : le foie chez le turbot, le foie et dans une moindre mesure le muscle chez la daurade, le tissu adipeux périviscéral et le muscle chez la truite. Chez les espè-

Tableau 1. L'augmentation du taux de lipides alimentaires affecte différemment la teneur en lipides des compartiments corporels selon les espèces.

\begin{tabular}{|c|c|c|c|c|c|c|}
\hline \multirow[b]{2}{*}{ Lipides aliment (\% matière sèche) } & \multicolumn{2}{|c|}{ Turbot $^{\mathrm{a}}$} & \multicolumn{2}{|c|}{ Daurade $^{b}$} & \multicolumn{2}{|c|}{ Truite arc-en-ciel } \\
\hline & 10 & 20 & 15 & 21 & 15 & 25 \\
\hline Foie & 13,8 & 21,1 & 10,9 & 15,8 & 5,2 & 7,4 \\
\hline Viscères & 1,6 & 1,9 & 33,6 & 36,5 & 51 & 59,5 \\
\hline Muscle & 0,6 & 0,6 & 7,6 & 8,9 & 8,8 & 11,2 \\
\hline
\end{tabular}

a d'après Régost et al. (2001).

${ }^{b}$ d'après Santinha et al. (1999).

c d'après Gélineau et al. (2001).

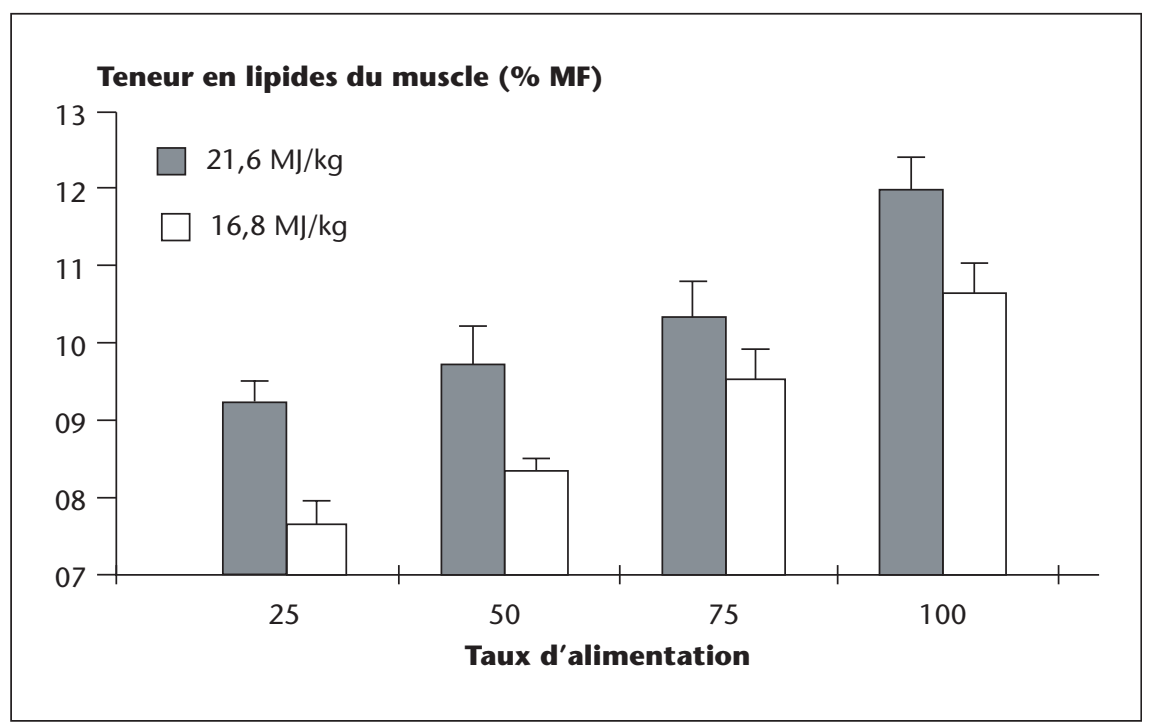

Figure 1. Effet du taux d'alimentation et du contenu énergétique de l'aliment sur la teneur en lipides du muscle de la truite arc-en-ciel $(1,8$ à 3,2 kg).

ces maigres comme la morue, le turbot, la sole, qui ont des capacités très limitées à stocker leurs réserves énergétiques sous forme de lipides dans le muscle, la teneur en lipides du muscle est peu sensible aux facteurs d'élevage (Regost et al., 2001 ; Jobling et al., 2008; Borges et al., 2009). En revanche, chez les espèces intermédiaires (truite, daurade, etc.) ou grasses (saumon, anguille, etc.), le compartiment lipides musculaires est d'une remarquable plasticité. La figure 1 illustre les variations de teneur en lipides du muscle de la truite arc-en-ciel en fonction du taux d'alimentation (25, 50, 75 ou $100 \%$ de la satiété) et en fonction du contenu énergétique de l'aliment (16,8 ou 21,6 MJ/kg aliment). Un changement de régime alimentaire pendant un à deux mois suffit pour obtenir des variations significatives de la teneur en lipides du muscle chez les salmonidés, indépendamment des variations de poids (Rasmussen, 2001). Les capacités maximales de stockage des lipides dans le compartiment musculaire des espèces intermédiaires et grasses ne sont pas clairement établies. Des résultats contradictoires sont parfois rap- portés. D'après certains auteurs (Einen et Skrede, 1998), la teneur en lipides du muscle du saumon atlantique n'augmente plus audelà d'un taux de lipides alimentaires de $34 \%$, suggérant que les capacités maximales de stockage des graisses dans le tissu musculaire sont atteintes. En revanche, d'autres auteurs (Hemre et Sandnes, 1999) rapportent que la teneur en lipides de la chair continue à augmenter avec un régime contenant $47 \%$ de lipides. Ces différences peuvent être liées à l'âge et la taille des poissons, la teneur en lipides du muscle augmentant avec le poids de l'animal, quelle que soit l'espèce (Shearer, 1994) ou à des variations individuelles d'origine génétique.

Ce sont les triglycérides de réserve qui sont responsables de la quasi-totalité de l'accroissement de la teneur en lipides observé dans le muscle suite à une augmentation de l'apport énergétique alimentaire, la teneur en phospholipides demeurant quasi inchangée (Takeuchi et al., 1978). Comme l'illustre le tableau 2, la proportion d'AGPI n-3 augmente avec la teneur en lipides du muscle, au détriment des AG mono-insaturés, la proportion d'AG saturés restant stable dans les lipides neutres comme dans les phospholipides (Kim et al., 1989). La composition en AG des phospholipides est affectée lorsque le taux de lipides de l'aliment passe de 9 à 13,5\% et reste stable au-delà. En revanche, la proportion d'AGPI n-3 des lipides neutres (triglycérides) du muscle augmente graduellement avec le taux de lipides de l'aliment, lorsque la source de lipides est de l'huile

Tableau 2. L'augmentation du taux de lipides alimentaires affecte la teneur en lipides du muscle et différemment les compartiments corporels selon les espèces (d'après Kim et al. (1989)).

\begin{tabular}{|c|c|c|c|}
\hline $\begin{array}{l}\text { Taux de lipides de } \\
\text { I'aliment (\% MS) }\end{array}$ & 9 & 13,5 & 20,5 \\
\hline Lipides muscle (\% MF) & 4,7 & 5,6 & 5,9 \\
\hline \multicolumn{4}{|l|}{$\begin{array}{l}\text { Acides gras lipides } \\
\text { neutres (\% AG) }\end{array}$} \\
\hline Saturés & 26,9 & 27,0 & 25,8 \\
\hline Mono-insaturés & 56,1 & 50,7 & 47,9 \\
\hline Polyinsaturés n-6 & 5,1 & 5,3 & 5,1 \\
\hline Polyinsaturés n-3 & 11,9 & 17,0 & 21,1 \\
\hline \multicolumn{4}{|l|}{$\begin{array}{l}\text { Acides gras } \\
\text { phospholipides (\% AG) }\end{array}$} \\
\hline Saturés & 27,2 & 28,4 & 28,6 \\
\hline Mono-insaturés & 29,3 & 21,5 & 23,6 \\
\hline Polyinsaturés n-6 & 5,0 & 5,1 & 3,5 \\
\hline Polyinsaturés n-3 & 38,5 & 45,0 & 44,3 \\
\hline
\end{tabular}


de poisson. Dans ce cas, plus la chair du poisson est grasse, plus elle apporte d'EPA et de DHA.

\section{Sélection génétique pour la teneur en lipides du muscle}

L'apport énergétique alimentaire, s'il permet de modifier la teneur en lipides du muscle de nombreuses espèces de poissons, affecte aussi d'autres compartiments corporels. Dans le cadre d'une collaboration interne à l'Inra, nous avons recherché la possibilité de modifier spécifiquement la teneur en lipides et en AGPILC n-3 de la chair des poissons par sélection génétique. La sélection génétique est une pratique relativement récente en aquaculture. Elle a eu comme premier objectif d'optimiser la vitesse de croissance des poissons. Cependant, les quelques résultats concernant le déterminisme génétique de l'engraissement, obtenus en particulier chez le saumon, suggéraient une héritabilité moyenne à haute $(0,2$ à 0,47$)$ pour la teneur en lipides musculaires (Gjerde et Schaeffer, 1989 ; Gjedrem, 1997).

Deux lignées expérimentales de truites arcen-ciel ont été créées, à I'Inra, par sélection divergente pour la teneur en lipides du muscle (Quillet et al., 2005). Les valeurs de teneurs en lipides et en EPA et en DHA du muscle des truites issues de la troisième génération de sélection divergente sont rapportées dans le tableau 3. Comme attendu, le taux de lipides du muscle est plus élevé pour la lignée muscle gras (+50\%) que pour la lignée muscle maigre (Kolditz et al., 2008), la sélection s'est donc avérée rapidement efficace. Cependant, la sélection génétique n'a pas modifié la composition en AG des lipides du muscle. Les proportions d'EPA et de DHA sont semblables dans le muscle des deux lignées (3,8 et $21,7 \%$ respectivement pour I'EPA et le DHA lorsque l'aliment contient $10 \%$ de lipides ; $6,4 \%$ d'EPA et $25,3 \%$ de DHA lorsque l'aliment contient $23 \%$ de lipides). En réponse à une augmentation du taux de lipides alimentaires, la teneur en lipides du muscle augmente chez les deux lignées (+52\% pour la lignée muscle maigre, $+59 \%$ pour la lignée muscle gras) ainsi que les proportions d'EPA et de DHA. Dans le cas présent, la proportion d'EPA a augmenté plus fortement que celle de DHA, car l'huile incorporée dans l'aliment était riche en EPA. En conséquence, la consommation de $100 \mathrm{~g}$ de chair de la lignée muscle gras apporte davantage d'EPA et de DHA que $100 \mathrm{~g}$ de chair de la lignée muscle maigre, uniquement parce que sa teneur en lipides est plus élevée. En revanche, la consommation de $100 \mathrm{~g}$ de chair de truite nourrie avec le régime à $23 \%$ de lipides apporte davantage d'EPA et de DHA que $100 \mathrm{~g}$ de chair de truite nourrie avec l'aliment à $10 \%$ de lipides à la fois parce que la chair est plus riche en lipides et parce que les proportions d'EPA et de DHA sont augmentées (tableau 3). La teneur en lipides du muscle peut donc être gérée à la fois par sélection génétique et par l'apport alimentaire ; en revanche, la nature des AG de la chair semble, jusqu'à présent, exclusivement contrôlée par l'apport alimentaire. Des travaux sont en cours pour rechercher d'éventuelles variations d'origine génétique de la teneur en AGPI-LC n-3 du muscle chez différentes familles de bars et de truites.

\section{Variation de la composition en AG de la chair des poissons en fonction des huiles du régime}

La composition en AG de la chair des poissons reflète assez fidèlement la composition en AG des lipides du régime alimentaire. On note d'ailleurs de grandes variations dans la composition en AG des lipides musculaires en fonction des préférences alimentaires des espèces. Alors que, chez les poissons carnivores (généralement espèces des eaux froides à tempérées), $75 \%$ en moyenne des AGPI sont de la série $\mathrm{n}-3$, chez les poissons herbivores ou omnivores comme le tilapia, le pangasius ou la carpe (espèces d'eau chaude ou tropicales), 60 à $80 \%$ des AGPI sont de la série n-6 (Médale, 2009). Lorsque ces animaux sont nourris avec des régimes contenant des huiles de poisson, la concentration en EPA et en DHA de leur chair augmente (tableau 4) (d'après Runge et al., 1987). De même, lorsque des poissons sont nourris avec des aliments contenant des huiles végétales, dépourvues d'EPA et de $\mathrm{DHA}$, en substitution de l'huile de poisson, la teneur en EPA et en DHA de leur chair diminue et celle des AG caractéristiques des huiles végétales incorporées augmente. Globalement, quelle que soit l'espèce, la réponse des $A G$ du muscle à l'apport alimentaire suit une simple loi de dilution, à l'exception du DHA (Robin et al., 2003 ; Jobling, 2004 ; Benedito-Palos et al., 2009). Grâce à la plasticité des lipides musculaires, le profil en AG caractéristique de I'huile de poisson peut être restauré en nourrissant les poissons avec un aliment à base d'huile de poisson durant les derniers mois précédant l'abattage, après un cycle d'élevage avec des aliments à base $d$ 'huiles végétales.

L'essor de l'aquaculture utilisant des aliments composés riches en lipides a accru fortement la demande en huile de poisson (Tacon et Metian, 2008). En parallèle, la stagnation des captures de pêche limite la disponibilité de cette matière première. L'aquaculture est confrontée aujourd'hui au double défi de réduire l'emploi de matières premières issues de la pêche pour l'alimentation des poissons d'élevage et de conserver à la chair de poisson la valeur nutritionnelle que lui confère sa richesse en AGPI-LC n-3. Différentes stratégies

Tableau 4. Composition en acides gras (pourcentage d'acides gras totaux) du muscle de la carpe en fonction de l'huile de l'aliment (d'après Runge et al. (1987)).

\begin{tabular}{|llll|}
\hline $\begin{array}{l}\text { Huile de } \\
\text { I'aliment }\end{array}$ & $\begin{array}{l}\text { Huile } \\
\text { de poisson }\end{array}$ & $\begin{array}{l}\text { Huile } \\
\text { de lin }\end{array}$ & $\begin{array}{l}\text { Huile } \\
\text { de maiis }\end{array}$ \\
\hline Saturés & 19,3 & 13,4 & 16,4 \\
\hline $\begin{array}{l}\text { Mono- } \\
\text { insaturés }\end{array}$ & 32,7 & 26,9 & 37,1 \\
\hline $18: 2 \mathrm{n}-6$ & 7,0 & 14,9 & 35,0 \\
\hline $18: 3 \mathrm{n}-3$ & 4,0 & 32,3 & 2,7 \\
\hline $20: 5 \mathrm{n}-3$ & 6,7 & 1,0 & 0,1 \\
\hline $22: 6 \mathrm{n}-3$ & 6,8 & 1,1 & 0,5 \\
\hline
\end{tabular}

Tableau 3. Effet de la sélection génétique et du contenu énergétique de l'aliment sur le taux de lipides, d'EPA et de DHA du muscle de la truite arc-en-ciel.

\begin{tabular}{|c|c|c|c|c|}
\hline \multirow[b]{2}{*}{ Taux de lipides de l'aliment } & \multicolumn{2}{|c|}{ Lignée muscle maigre } & \multicolumn{2}{|c|}{ Lignée muscle gras } \\
\hline & 10 & 23 & 10 & 23 \\
\hline \multicolumn{5}{|l|}{ Lipides du muscle } \\
\hline Pourcentage de poids frais & 4,2 & 6,4 & 6,3 & 10 \\
\hline \multicolumn{5}{|l|}{ EPA } \\
\hline Pourcentage d'AG totaux & 3,7 & 6,6 & 3,9 & 6,3 \\
\hline $\mathrm{g} / 100 \mathrm{~g}$ chair & 0,15 & 0,40 & 0,23 & 0,59 \\
\hline \multicolumn{5}{|l|}{ DHA } \\
\hline Pourcentage d'AG totaux & 21,2 & 25,3 & 22,3 & 24,5 \\
\hline $\mathrm{g} / 100 \mathrm{~g}$ chair & 0,85 & 2,16 & 1,31 & 2,40 \\
\hline
\end{tabular}


ont été explorées : choix de différentes huiles de substitution seules ou en mélange, remplacement partiel pendant le cycle d'élevage, alimentation dite « de finition » dans les mois précédant la commercialisation, durée de cette phase de finition et choix des huiles de poissons utilisées. Parmi les alternatives aux huiles extraites des poissons issus de la pêche, seules les huiles végétales sont produites en quantités suffisantes pour répondre à la demande de l'aquaculture, les quantités de lipides provenant des déchets des pêches ou de l'industrie de transformation des poissons, du zooplancton ou d'algues étant actuellement faibles. Les essais de substitution de l'huile de poisson ont, en majorité, été conduits avec des huiles de soja, de palme, de colza et de tournesol qui représentent environ $80 \%$ de la production mondiale d'huiles végétales ainsi qu'avec de I'huile de lin, qui présente l'avantage d'être riche en C18:3 n-3, précurseur de l'EPA et du $\mathrm{DHA}$. Les différentes études montrent que le remplacement de l'huile de poisson par une ou des huiles végétales ne modifie pas la croissance des poissons et la teneur en lipides de leur chair à partir du moment où leurs besoins en AGPI n-3 sont couverts. Les poissons marins sont incapables de synthétiser I'EPA et le DHA, ils doivent trouver ces AG dans I'aliment pour couvrir leurs besoins. Chez les poissons d'eau douce, le $\mathrm{C} 18: 3 \mathrm{n}-3$ peut suffire à couvrir les besoins, mais sa conversion en EPA et en DHA étant limitée, un apport direct d'EPA et de DHA par l'aliment est plus efficace. L'inconvénient majeur du remplacement de l'huile de poisson par des huiles végétales dans les aliments piscicoles est donc le changement de composition en acides de la chair. Ces changements sont d'autant plus marqués que le taux de substitution est élevé (Benedito-Palos et al., 2009 ; Bell et al., 2003a, 2004). L'enjeu des recherches actuelles est de trouver des solutions pour minimiser l'impact négatif des huiles végétales sur le profil en AG de la chair des poissons tout en épargnant I'huile de poisson. L'emploi d'un mélange d'huiles est préférable à un substitut unique. Pour maintenir le rapport n-3/n-6 de la chair aussi élevé que possible, il faut :

- limiter l'apport de C18:2 n-6 qui est faiblement catabolisé par les poissons pour produire de l'énergie et qui s'accumule dans le muscle ; - privilégier l'apport d'AG mono-insaturés, car ils sont efficacement utilisés comme source $d^{\prime}$ énergie et permettent de limiter la proportion de C18:2 n-6 dans I'aliment ;

- apporter du C18:3 qui, même s'il est faiblement (poissons d'eau douce) ou pas (poissons marins) converti en EPA et en DHA, permet $d^{\prime}$ augmenter le rapport $n-3 / n-6$ et contribue à la qualité nutritionnelle de la chair du poisson. Le but de l'alimentation de "finition » à base $d$ 'huile de poisson est de restaurer des teneurs élevées en EPA et en DHA et de diminuer le contenu en C18:2 n-6. Chez le saumon ayant été nourri pendant 50 semaines (de $120 \mathrm{~g}$ à $2,3 \mathrm{~kg}$ ) avec un aliment contenant de I'huile de colza comme seule source de lipide, une alimentation à base $\mathrm{d}^{\prime}$ huile de poisson pendant 20 semaines (2,3 à 3,5 kg) permet de restaurer $90 \%$ des teneurs en EPA et en DHA mais n'élimine que $50 \%$ du C18:2 n-6 accumulé (Bell et al., 2003b). Dans une autre étude avec des saumons de 200 à $500 \mathrm{~g}$, les mêmes auteurs (Bell et al., 2003a) montrent que la teneur en EPA est rétablie plus rapidement (quatre semaines) que celle en DHA. La durée d'alimentation de finition nécessaire dépend aussi de l'espèce et de l'huile de poisson employée. Elle est plus courte chez des espèces dont le muscle est moins riche en lipides que celui du saumon. Parmi les huiles de poisson, les huiles d'anchois, de sardine et de menhaden sont plus efficaces que les huiles de capelan ou de maquereau, car elles sont plus concentrées en AGPI-LC n-3. Des travaux sont encore nécessaires pour définir les conditions les plus favorables pour réduire le plus possible l'emploi d'huile de poisson tout en optimisant la composition en AG de la chair des différentes espèces de poissons d'élevage.

\section{Protection contre la peroxydation par apport alimentaire de composés antioxydants}

Les lipides de la chair des poissons peuvent être facilement dégradés, car les doubles liaisons des AGPI sont particulièrement sensibles au phénomène de peroxydation. En effet, les ris- ques de dégradation par des réactions radicalaires en chaîne augmentent avec le degré $d$ 'insaturation des AG (Crockett, 2008). La peroxydation des AG insaturés, qui se produit lors de l'exposition à l'air et à la lumière (conditions rencontrées sur les étals) conduit à la formation d'aldéhydes, de cétones et de radicaux libres. Elle diminue la valeur nutritionnelle des lipides en réduisant la quantité d'AGPI-LC et dégrade les caractéristiques sensorielles du produit en provoquant l'apparition d'odeurs désagréables et parfois l'altération de la couleur (Frigg et al., 1990 ; Tocher et al., 2002, 2003). Des mesures réalisées in vitro avec des explants de muscle de truite et de bar contenant différents taux de lipides montrent que la quantité de produits oxydés formés augmente avec la teneur en lipides du muscle et sa concentration en AGPI-LC (Alvarez et al., 1998). Des phénomènes semblables sont observés in vivo (Tocher et al., 2002).

L'apport alimentaire de composés antioxydants permet de protéger les AG longs de la peroxydation, préservant ainsi la valeur nutritionnelle de la chair des poissons. Plusieurs études conduites chez différentes espèces de poissons ont démontré l'efficacité des tocophérols et en particulier de l' $\alpha$-tocophérol (vitamine E) pour prévenir la peroxydation des lipides par des réactions radicalaires et réduire ainsi la formation des composés issus de ces réactions tels que les malonaldéhydes (Frigg et al., 1990 ; Tocher et al., 2003). La teneur en vitamine $\mathrm{E}$ du muscle de poisson augmente avec le taux de vitamine E de l'aliment (figure 2). La concentration en malonaldéhydes, issus de l'oxydation des lipides, est d'autant plus faible que la chair est riche en vitamine $\mathrm{E}$ (Frigg et al., 1990 ; Tocher et al., 2002). Comme l'illustre la figure 2,

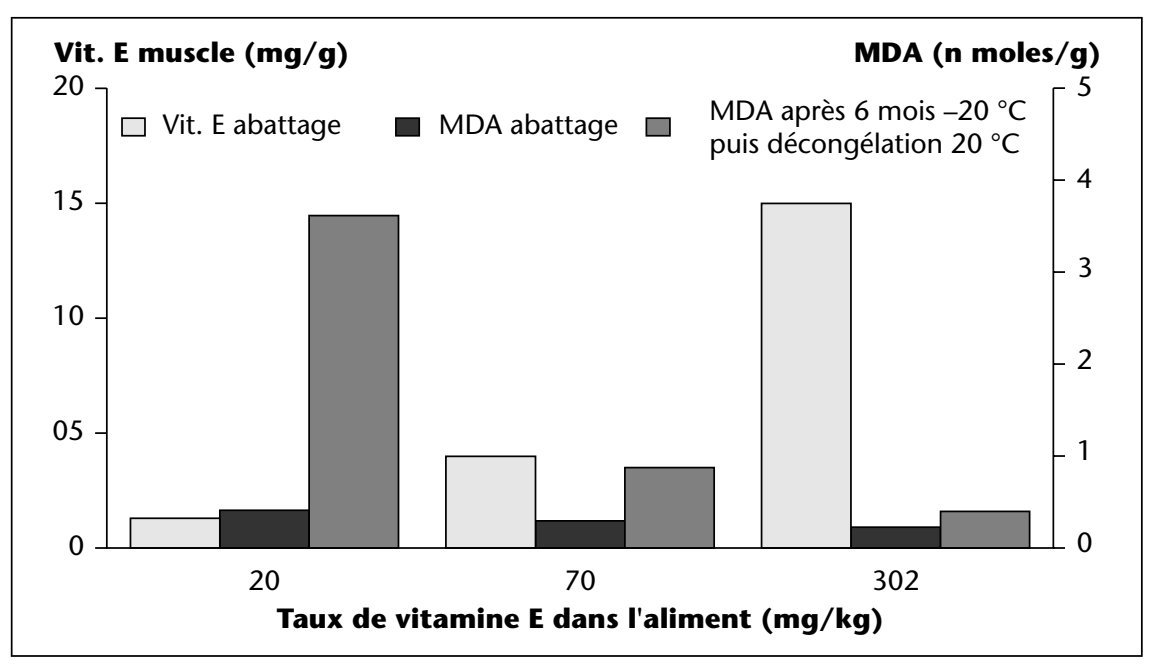

Figure 2. Conséquences du taux de vitamine E de l'aliment sur la teneur en vitamine E dans le muscle de turbot et sa teneur en malonaldéhyde (MDA) dans la chair au moment de l'abattage et après six mois de congélation à $-20{ }^{\circ} \mathrm{C}$ (d'après Stéphan et al. (1995)). 
des teneurs élevées en vitamine $E$ protègent aussi des phénomènes de peroxydation des lipides qui peuvent se produire pendant le stockage et lors de la décongélation du produit, la formation des composés peroxydés est inhibée. La vitamine $\mathrm{E}$ contribue ainsi à la conservation des qualités nutritionnelles du produit en préservant I'intégrité des AG (Stéphan et al., 1995).

\section{Conclusion}

Les pratiques d'élevage, en particulier la sélection génétique et l'alimentation permettent donc de moduler la quantité de lipides de la chair des poissons, dans les limites permises par l'espèce. La quantité d'AG longs polyinsaturés $n-3$ peut être modifiée soit indirectement via la teneur en lipides du filet, soit directement par les huiles contenues dans I'aliment. L'adjonction de substances antioxydantes, telles que la vitamine $E$ dans les aliments, permet de prévenir la dégradation des AG longs polyinsaturés par peroxydation, contribuant ainsi à conserver leur valeur nutritionnelle. Les recherches en cours ont pour objectifs de valoriser au mieux les huiles de poisson, de chercher des substituts efficaces pour maintenir des teneurs élevées en AGPI-LC n-3 dans la chair des poissons d'élevage et d'évaluer la possibilité de sélectionner des familles présentant des capacités particulières pour le stockage d'EPA et de DHA.

\section{RÉFÉRENCES}

Alvarez MJ, Lopez-Bote Cl, Diez A, et al. Dietary fish oil and digestible protein modify susceptibility to lipid peroxidation in the muscle of rainbow trout (Oncorhynchus mykiss) and sea bass (Dicentrarchus labrax). Brit J Nut $1998 ; 80: 281-9$.

Bell JG, McGhee F, Campbell PJ, Sargent JR. Rapeseed oil as an alternative to marine fish oil in diets of postsmolt Atlantic salmon (Salmo salar): changes in flesh fatty acid composition and effectiveness of subsequent fish oil "wash out". Aquaculture 2003a ; 218 : 515-28.

Bell JG, Tocher DR, Henderson RJ, Dick JR, Crampton VO. Altered fatty acid compositions in Atlantic salmon (Salmo salar) fed diets containing linseed and rapeseed oils can be partially restored by a subsequent fish oil finishing diet. J Nutr 2003b; 133 : 2793-801.

Bell JG, Henderson RJ, Tocher DR, Sargent JR. Replacement of dietary fish oil with increasing levels of linseed oil: modification of flesh fatty acid compositions in Atlantic salmon (Salmo salar) using a fish oil finishing diet. Lipids 2004 ; 39 : 223-32.
Benedito-Palos L, Navarro JC, Bermejo-Nogales A, Saera-Vila A, Kaushik S, Pérez-Sánchez J. The time course of fish oil wash-out follows a simple dilution model in gilthead sea bream (Sparus aurata L.) fed graded levels of vegetable oils. Aquaculture 2009 ; 288 : 98-105.

Borges P, Oliveira B, Casal S, Dias J, Conceicao L, Valente LMP. Dietary lipid level affects growth performance and nutrient utilisation of Senegalese sole (Solea senegalensis) juveniles. Brit / Nut 2009; 102 : 1007-14.

Corraze G, Kaushik SJ. Les lipides des poissons marins et d'eau douce. OCL $1999 ; 6: 111-5$.

Crockett EL. The cold but not hard fats in ectotherms: consequences of lipid restructuring on susceptibility of biological membranes to peroxidation, a review. J Comp Physiol B 2008 ; 178 : 795-809.

Einen O, Skrede G. Quality characteristics in raw and smoked fillets of Atlantic salmon, Salmo salar, fed high-energy diets. Aquac Nutr 1998 ; 4 : 99-108.

FAO State of fisheries and aquaculture 2008. Food and Agriculture organization of the united nations, Rome, 2009.

Frigg M, Prabucki AL, Rudhel EU. Effect of dietary vitamin E levels on oxydative stability of trout filets. Aquaculture $1990 ; 84: 145-58$.

Gélineau A, Corraze G, Boujard T, Larroquet L, Kaushik $S$. Relation between dietary lipid level and voluntary feed intake, growth, nutrient gain, lipid deposition and hepatic lipogenesis in rainbow trout. Reprod Nutr Dev 2001 ; 41 : 487-503.

Gjedrem T. Flesh quality improvement in fish trough breeding. Aquac Int 1997 ; 5 : 197-206.

Gjerde B, Schaeffer LR. Body traits in rainbow trout. II. Estimates of heritabilities and of phenotypic and genetic correlations. Aquaculture $1989 ; 80$ : 25-44.

Hemre Gl, Sandnes K. Effect of dietary lipid level on muscle composition in Atlantic salmon Salmo salar. Aquac Nutr $1999 ; 5$ : 9-16.

Henderson RJ, Tocher DR. The lipid composition and biochemistry of freshwater fish. Prog Lipid Res 1987 ; $26: 281-347$

Kim JD, Kaushik SJ, Pascaud M. Effects of dietary lipid to protein ratios on the fatty acid composition of muscle lipids in rainbow trout. Nut Rep Internat $1989 ; 40: 9-16$.

Kolditz C, Borthaire M, Richard N, et al. Liver and muscle metabolic changes induced by dietary energy content and genetic selection in rainbow trout (Oncorhynchus mykiss). Am J Physiol 2008; 294 : R1154-R1R64.

Jobling M. Are modifications in tissue fatty acid profiles following a change in diet the result of dilution? Test of a simple dilution model. Aquaculture 2004 ; $232: 551-62$.
Jobling M, Leknes O, Saether BS, Bendiksen EA. Lipid and fatty acid dynamics in Atlantic cod, Gadus morhua, tissues: influence of dietary lipid concentrations and feed oil sources. Aquaculture 2008 ; 281 : 87-94.

Médale F. Teneur en lipides et composition en acides gras de la chair de poissons issus de la pêche et de l'élevage. Cah Nutr Diet 2009 ; 44 : 173-81.

Quillet E, Le Guillou S, Aubin J, Fauconneau B. Twoway selection for muscle lipid content in pan-size rainbow trout (Oncorhynchus mykiss). Aquaculture $2005 ; 245: 49-61$.

Rasmussen RS. Quality of farmed salmonids with emphasis on proximate composition, yield and sensory characteristics. Aquac Res 2001 ; 32 : 767-86.

Regost C, Arzel J, Cardinal M, Robin J, Laroche M, Kaushik S). Dietary lipid level, hepatic lipogenesis and flesh quality in turbot (Psetta maxima). Aquaculture $2001 ; 193$ : 291-309.

Robin JH, Regost C, Arzel J, Kaushik SJ. Fatty acid profile of fish following a change in dietary fatty acid source: model of fatty acid composition with a dilution hypothesis. Aquaculture 2003 ; 225 : 283-93.

Runge G, Steinhart H, Schwarz F), Kirchgessner M. Influence of different fats with varying additions of alpha-tocopheryl acetate on the fatty acid composition of carp (Cyprinus carpio L.). Fat Sci Technol 1987 ; 89 : 389-93.

Santinha PJM, Médale F, Corraze G, Gomes EFS. Effects of the dietary protein/lipid ratio on growth and nutrient utilization in gilthead seabream (Sparus aurata L.). Aquac Nutr $1999 ; 5$ : 147-56.

Shearer K. Factors affecting the proximate composition of cultured fishes with emphasis on salmonids. Aquaculture $1994 ; 119$ : 63-88.

Stéphan G, Guillaume J, Lamour F. Lipid peroxidation in turbot (Scophthalmus maximus) tissue: Effect of dietary vitamin $\mathrm{E}$ and dietary $\mathrm{n}-6$ or $\mathrm{n}-3$ polyunsaturated fatty acids. Aquaculture $1995 ; 130$ : 251-68.

Tacon AG), Metian M. Global overview on the use of fish meal and fish oil in industrially compounded aquafeeds: Trends and future prospects. Aquaculture $2008 ; 285$ : 146-58.

Takeuchi T, Watanabe T, Ogino C. Supplementary effects of lipids in a high protein diet for rainbow trout. Bull Japan Soc Sci Fish 1978 ; 44 : 677-81.

Tocher DR, Mourente G, Van der Eecken A, et al. Effects of dietary vitamin $E$ on antioxidant defence mechanisms of juvenile turbot (Scophthalmus maximus L.), halibut (Hippoglossus hippoglossus L.) and sea bream (Sparus aurata L.). Aquac Nutr 2002 ; 8 : 195-207.

Tocher DR, Mourente G, Van der Eecken A, et al. Comparative study of antioxidant defence mechanisms in marine fish fed variable levels of oxidised oil and vitamin E. Aquac Int 2003 ; 11 : 195-216. 